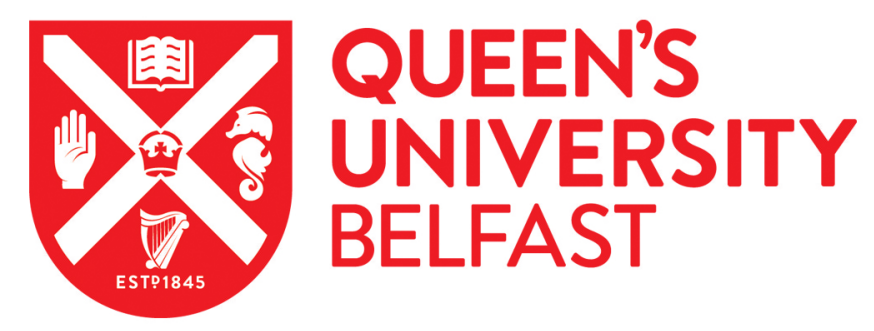

\title{
A microscale integrated approach to measure and model fibre misalignment in fibre-reinforced composites
}

Sebaey, T. A., Catalanotti, G., \& O'Dowd, N. P. (2019). A microscale integrated approach to measure and model fibre misalignment in fibre-reinforced composites. Composites Science and Technology, 183, [107793].

https://doi.org/10.1016/j.compscitech.2019.107793

\section{Published in:}

Composites Science and Technology

Document Version:

Peer reviewed version

Queen's University Belfast - Research Portal:

Link to publication record in Queen's University Belfast Research Portal

\section{Publisher rights}

Copyright 2019 Elsevier

This manuscript is distributed under a Creative Commons Attribution-NonCommercial-NoDerivs License

(https://creativecommons.org/licenses/by-nc-nd/4.0/), which permits distribution and reproduction for non-commercial purposes, provided the author and source are cited.

\section{General rights}

Copyright for the publications made accessible via the Queen's University Belfast Research Portal is retained by the author(s) and / or other copyright owners and it is a condition of accessing these publications that users recognise and abide by the legal requirements associated with these rights.

Take down policy

The Research Portal is Queen's institutional repository that provides access to Queen's research output. Every effort has been made to ensure that content in the Research Portal does not infringe any person's rights, or applicable UK laws. If you discover content in the Research Portal that you believe breaches copyright or violates any law, please contact openaccess@qub.ac.uk. 


\title{
A microscale integrated approach to measure and model fibre misalignment in fibre-reinforced composites
}

\author{
T.A. Sebaey ${ }^{a, b, *}$, G. Catalanotti ${ }^{c}$, N.P. O'Dowd ${ }^{a}$ \\ ${ }^{a}$ School of Engineering, Irish Composites Centre (IComp), Bernal Institute, University of Limerick, \\ Limerick, Ireland. \\ ${ }^{b}$ Engineering Management Department, College of Engineering, Prince Sultan University, Riyadh, \\ Saudi Arabia \\ ${ }^{c}$ Advanced Composites Research Group (ACRG), School of Mechanical and Aerospace Engineering, \\ Queen's University Belfast, Belfast, United Kingdom
}

\begin{abstract}
Computational micromechanics of fibre-reinforced polymers (FRPs) relies on the ability of the representative volume elements (RVEs) to take into account the different features that characterise the geometry of the material system under consideration. Fibre misalignment has been proven experimentally to have a significant effect on the mechanical properties at the macroscale, but is not currently taken into consideration in models at the individual fibre level, perhaps due to the difficulty in statistically characterising the fibre misalignment. In this work, an integrated approach is presented to measure and model fibre misalignments in FRPs. A computed tomography (CT) scan is used to identify the fibre geometry and statistically characterise the fibre misalignment angle distribution. Using a methodology recently developed by the authors, three-dimensional (3D) RVEs were generated by requiring their misalignment angle distribution to fit the empirical distribution. The methodology proposed provides a framework for the systematic numerical analysis of the influence of fibre misalignment on mechanical properties of FRPs.

Keywords:
\end{abstract}

\footnotetext{
*Tamer A. Sebaey is on leave from Mechanical Design and Production Engineering Dept., Faculty of Engineering, Zagazig University, Sharkia, Egypt.

Email addresses: sepaey@hotmail.com (T.A. Sebaey), g.catalanotti@qub.ac.uk (G. Catalanotti), noel.odowd@ul.ie (N.P. O'Dowd)
} 
A. Carbon fibre composites; C. Statistics; C. Modelling; D. X-ray diffraction; Fibre misalignment

\section{Introduction}

In existing micromechanical models of fibre reinforced polymers (FRPs), all fibres are assumed parallel and in a well-defined direction, e.g. [1,2]. This is generally not the case in real composites, due to the nature of the manufacturing process and the thermal residual stress developed during curing [3,4]. Different fibre bundles (thousands of continuous individual fibre filaments laid together) tend to have a misalignment with their neighbouring bundles and even within the same bundle, fibres are generally not perfectly parallel and aligned [5]. The presence of misoriented fibres tends to promote microbuckling [6], which is the main damage mechanism in uniaxial compression of FRPs. As a result, fibre misalignment is expected to have a significant influence on mechanical properties, such as longitudinal compression strength and flexural strength. The compressive strength represents one of the limiting design factors in large composites structures [7] and it is thus important to accurately represent this failure mode in models of composite failure.

Fibre misalignment can be classified, based on the size scale, as either ply misalignment or individual fibre misalignment. Ply misalignment (usually referred to in the literature as fibre/ply waviness or wrinkling) can be easily noted in the out-of-plane direction and is usually assumed to be in a well-defined form (sinusoidal, etc.). For a multidirectional laminate under general loading conditions, Fedulov et al. [8] showed using finite element analysis that the presence of ply misalignment can lead to a reduction of up to $49 \%$ in the laminate compressive strength. This effect was confirmed experimentally, by Wilhelmsson et al. [9], who demonstrated that for CFRP laminates with a misalignment (on the ply level) of $6^{\circ}$, the compressive strength is reduced by $50 \%$. Davidson et al. [10] studied the effect of a controlled ply waviness on compression strength by inserting pre-cured resin blocks at the centre of the specimen during manufacture, resulting in misalignment angles ranging from from $10^{\circ}$ to $35^{\circ}$. The results 
showed a reduction of $50 \%$ of the compressive strength within the range of studied misalignment angles. It is worth remarking that the range used in Davidson et al.'s study is high compared to typical manufacturing-induced misalignment angles [11, 12]. Artificially introduced waviness/misalignment was also studied experimentally by Raghavalu Thirumalai et al. [13] with a measured reduction in strength and stiffness for both tension and compression loadings. The effect of introducing artificially induced fibrewrinkling on the compression strength was investigated experimentally and numerically by Mukhopadhyay et al. [14]. The analysis showed a high stress concentration at the wrinkling area, which initiated early damage and resulted in strength reduction. Larranaga-Valsero et al. [15] measured controlled wrinkling in glass-carbon hybrid laminates using ultrasound techniques. High reductions (up to 63\%) in the compression and tensile strength were recorded.

Theoretical efforts, to consider the fibre misalignment in FRP, are recognized in the literature $[16,17]$. Argon [18] presented a model to predict the compression strength of unidirectional composites as a function of shear strength and ply misalignment angle. This model shows the major role that ply misalignment plays in predicting compressive strength of FRPs. Zhu et al. [19] studied, analytically, the effect of ply waviness on the predicted elastic constants using classical laminate theory. The waviness pattern, the length of the waviness zone and the off-axis angle showed significant effects on the predicted elastic constants. Takeda [20] presented a two-step homogenization technique, based on mixed boundary conditions, in order to predict the three-dimensional effective elastic properties of wrinkled laminates. The model was applied to several CFRP laminates with uniform and graded ply waviness and good agreement with the experimentally measured properties were achieved.

In [21] an ultrasonic array was used to assess the in-plane and out-of-plane waviness in several composite systems and was shown to be effective at measuring ply level misorientation. At the individual fibre scale, misalignment can occur in both in-plane and out-of-plane directions. Hillig [5] measured fibre misalignment by cutting the sample perpendicular to the fibre direction and analysing the ellipse resulting from the projec- 
tion of each individual fibre. The analysis assumed that the fibres in each fibre bundle are parallel and the bundles are not parallel to each another. To extend the measurements along the fibre direction, Clarke et al. [22] followed the approach in [5], carrying out measurements at different depths using through thickness laser scanning, and constructing a composite image to obtain 3D information about each fibre. The method in [22] is cumbersome and is limited to a small number of fibres at a few locations within the material. Creighton et al. [6] scanned specimens in a direction parallel to the fibre direction using optical microscopy and analysed the images by measuring the angles of different fibres at different locations. This procedure provides more robust information about fibre misalignment, but it still based on 2D planar information and is destructive. A similar but more time efficient and automated procedure was reported by Kratmann et al. [23], using images obtained from optical microscopy. The method assumed that each fibre is a straight line with a well-defined orientation measured as the average of the orientations measured along the fibre length. Mizukami et al. [24] used an eddy current technique to measure fibre waviness in ultra-thin highly aligned layers. Requena et al. [25] used computed tomography (CT) to measure fibre orientation assuming that each fibre has a single orientation and, moreover, that a fibre bundle could be described with a single orientation which is the mean of the individual orientations of the fibres. Sutcliffe et al. [26] modified the method in [6] to analysis the images obtained using both optical and CT scans. The results showed that the alignment of fibres in prepreg composites is better than that from composites manufactured using resin transfer moulding (RTM). More recently, Wilhelmsson and Asp [27] presented a methodology to measure fibre misalignment, based on the analysis of optical micrographs, by tracking the footprint of each individual fibres within a predefined cell.

The research available in the literature to date focuses on either the assessment of misalignment (see for example $[25,26]$ ) or on the effect of the misalignment on mechanical properties (see for example [14, 28]). There are some, limited computational studies on misalignment at the ply level (see for example $[8,29]$ ). However, to our knowledge there is no work to date in the literature which examines how to represent fibre mis- 
alignment statistically and how to model this measured misalignment at the fibre level. This work aims to fill this gap. The approach is as follows: deviations in fibre angles are first determined by a CT scan, allowing the footprint of individual fibres to be tracked. Next, the data are analysed statistically, and fitted using the von Mises distribution, to obtain the concentration parameter, $\kappa$, which quantifies the level of mis-orientation. A 3D representative volume element (RVE) with the same concentration parameter, $\kappa$, is then constructed following the procedure proposed in [30], in order to make the misalignment in the RVE statistically equivalent to that measured experimentally.

\section{Experimental Procedure}

\subsection{Materials and Manufacturing System}

Two material systems are examined in this investigation. The first is IM7/8552, a typical thermoset composite used in the aerospace industry. The second is IM7/PEEK, which is a thermoplastic material. Both systems share the same fibre type (Hexcel Hextow@IM7 carbon fibre), have nominal fibre volume fraction of $60 \%$, and are manufactured using pre-preg, with the same stacking sequence, $\left[0_{2} / 90_{4}\right]$. This stacking sequence allows easy access to both longitudinal and transverse directions. The two materials are subjected to different manufacturing processes/techniques that affects the degree of fibre alignment, allowing different ranges of fibre misalignment to be examined. The thermoset based composite material (IM7/8552) was manufactured using the standard autoclave cycle with maximum pressure of 7 bar and maximum temperature of $180^{\circ} \mathrm{C}$. Square sheets of prepreg were laid in the desired stacking sequence and placed in the autoclave. The thermoplastic material (IM7/PEEK) was manufactured by automated tape placement (ATP), using a laser-assisted tape placement head attached to a robot arm. The ATP process is accomplished by placing the pre-preg tape in place using the robot arm; at the same time, the laser melts, in-situ, the portion of the tape ready to be laid (laser temperature is $410^{\circ} \mathrm{C}$ ) and a silicone consolidation roller applies pressure and cooling to finalise the process. The roller used in the current study was 
pressed onto the substrate by a pneumatic cylinder and cooled externally using compressed air to prevent the tape adhering to the roller during processing. Further details on the ATP process and the control parameters are in [31, 32].

The resulting laminates have thicknesses of $1.50^{ \pm 0.07} \mathrm{~mm}$ and $1.796^{ \pm 0.13} \mathrm{~mm}$ for the IM7/8552 and the IM7/PEEK, respectively. After curing, $30 \mathrm{~mm}$ was trimmed from all laminate edges. Trimming and other cutting were performed using a computer controlled diamond cutter with a hydraulic specimen holding mechanism. Slices of the laminates with dimensions $70 \mathrm{~mm} \times 2 \mathrm{~mm}$ were cut for CT scanning.

\subsection{Computed tomography (CT) scans}

The specimens were mounted vertically inside the CT scanner (a Zeiss Xradia 510 Versa instrument). Three specimens were used for each material system and three scans were performed for each specimen (in total, nine scans per material system were considered). For each specimen, the three scans were taken at the two ends and the middle of the specimen. The X-ray voltage and current were $80 \mathrm{kV}$ and $87 \mu \mathrm{A}$, respectively. The pixel size was $0.36 \mu \mathrm{m}$ and each individual image was $924 \times 924$ pixels. The resulting field of view is a cylinder of $300 \mu \mathrm{m}$ diameter and $300 \mu \mathrm{m}$ length. In total, 3201 projections were collected from each scan, with exposure time $5 \mathrm{~s}$, and a sample rotation of $360^{\circ}$, resulting in a total scan time of $4.5 \mathrm{~h}$. The result of this step is a series of images (hundreds of images) through the specimen cross-section. The images are then reconstructed into a 3D shape using ImageJ software [33]. 3D images of typical scans, after processing by ImageJ, are shown in Figure 1 for the two material systems. In addition to the fibre misalignments, the CT scan of the IM7/PEEK material (Figure 1(b)) shows features not typically seen in thermoset composites, such as interlaminar and intralaminar voids.

To assess fibre misalignment, sections were taken from the 3D image along the fibre length. To consider both in-plane and the out-of-plane fibre misalignments, horizontal and vertical cuts were considered, see Figure 1(a). The resulting 2D shapes are 0.3 $\mathrm{mm}$ in length with varying height, depending on the section. A portion of a sample 


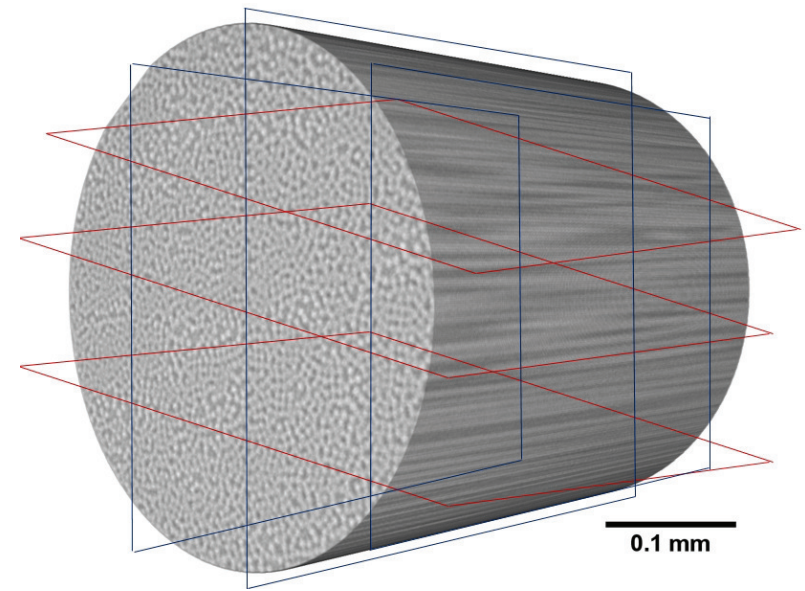

(a) IM7/8552

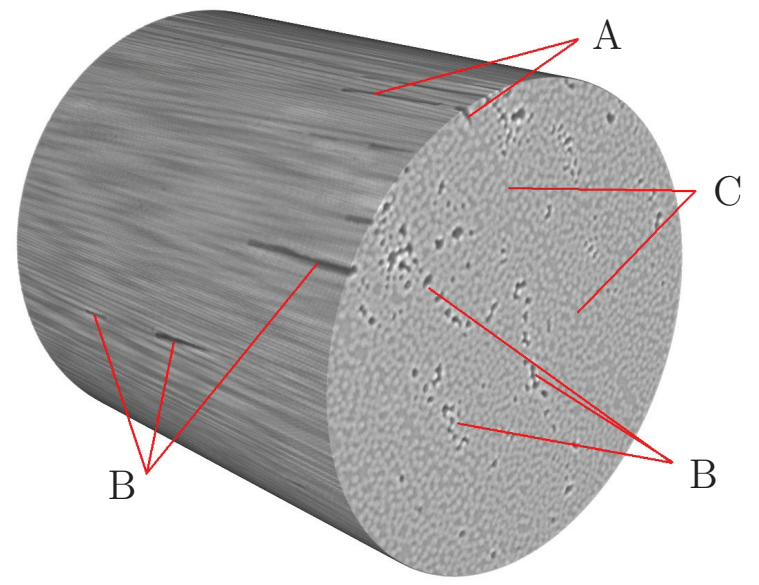

(b) IM7/PEEK

Figure 1: Sample CT scans. In (a), the red planes represent the in-plane direction and the blue ones the out-of-plane directions. In (b), A: Interlaminar voids; B: Intralaminar voids; C: Boundaries between layers.

image is shown in Figure 2(a). The image is first processed by phase separation in ImageJ, based on the pixel colour, to leave only the fibre. Straight line segments are then superimposed on the fibres (see Figure 2(b) for illustration). The lines follow the centre of the individual fibres, with a length of 30-50 $\mu \mathrm{m}$. The length of each line (which defines the number of lines per each fibre) is defined based on the degree of straightness of the individual fibre, i.e., fibres with more misalignments require greater number of short lines to be represented correctly. The deviation of each line from the horizontal is then automatically measured. For each 2D image, 200 measurements are taken and for each specimen, a minimum of 4,000 measurements are considered. The current study assumes that fibre misalignments in the in-plane and out-of-plane directions are equivalent, as we could not identify any differences in the measurements taken in both directions.

\section{Statistical analysis}

The data generated as described in Section 2.2 contains a set of angular deviations (in degrees) for each material system. Jelf and Fleck [34] and Bednarcyk et al. [17] have shown that fibre misalignment angles in composites can be reasonably approximated 


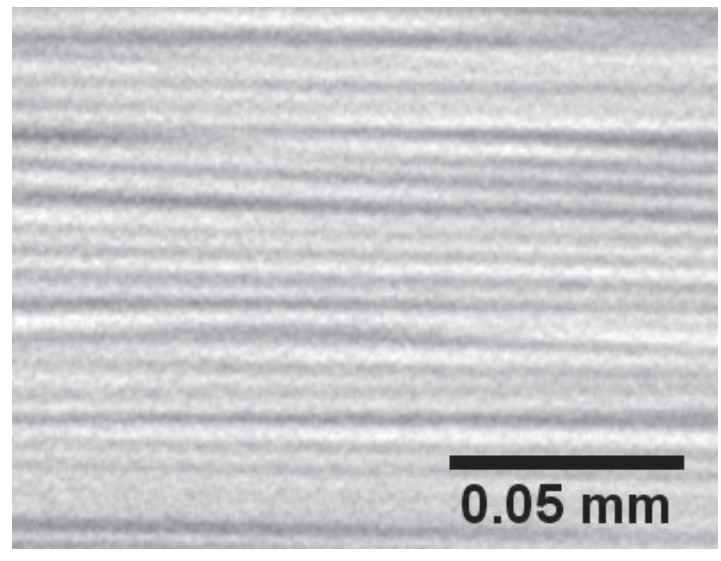

(a) Raw image

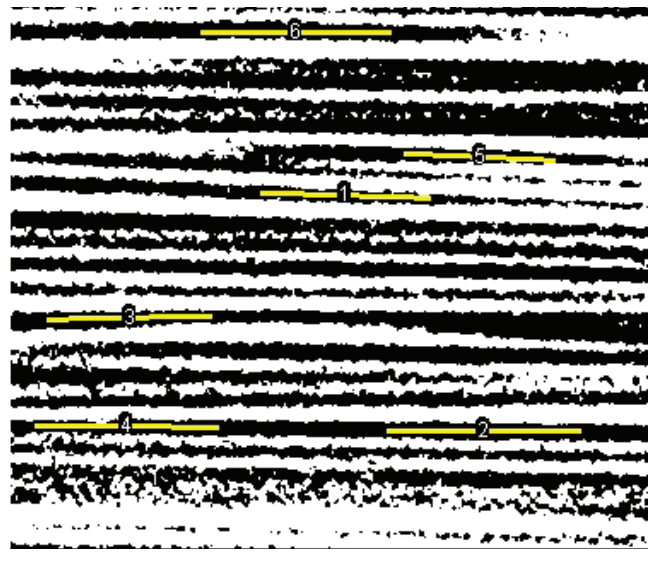

(b) processed image

Figure 2: an example of processing the 2D sections obtained from the $3 \mathrm{D}$ shape. The fibres are the black regions in the processed image (b)

by a normal distribution. Considering their angular nature, such readings should be treated using circular statistics $[35,36]$. The von Mises distribution is a close approximation to the wrapped normal distribution, which is the circular analogue of the normal distribution and has previously been used to represent fibre misalignments, [37], [28]. The von Mises probability density function $P$ is given by:

$$
P(\alpha, \mu, \kappa)=\frac{1}{2 \cdot \pi \cdot I_{0}(\kappa)} \cdot e^{(\kappa \cdot \cos \alpha-\mu)},
$$

where $\alpha$ is the parameter being analysed, in our case the deviation from the designated fibre orientation (in radians), $\mu$ is a measure of location, the distribution is clustered around $\mu$ which is equivalent to the mean of the normal distribution, and $\kappa$ is a concentration parameter, a reciprocal measure of dispersion, so $1 / \kappa$ is analogous to the variance in the normal distribution. $I_{0}$ in Equation 1 is the modified Bessel function of order 0 . If $\kappa$ is zero, the von Mises distribution, $P(\alpha, \nu, \kappa)$ is uniform, and for small $\kappa$, the distribution is close to uniform. If $\kappa$ is large, the distribution becomes concentrated about the angle $\mu$ with $\kappa$ being a measure of the concentration. In our case, the larger the value of $\kappa$, the closer the fibres are to the designated angle, which represents a good alignment of fibres. For a given data set of misalignment angle, $\alpha$, the concentration 
parameter, $\kappa$, can be estimated by computing the maximum likelihood. A detailed algorithm to estimate $\kappa$ for a given set of data can be found in [38].

\section{Generation of the RVEs}

The general procedure for the generation of the RVE was proposed in [30] and it is summarized here for the sake of completeness. In order to obtain a 3D RVE with fibre misalignments, the aforementioned procedure requires three steps: (i) the generation of the 2D distribution, (ii) the generation of a perturbed 3D RVE, and (iii) the optimisation of the perturbed RVE in order to minimise the error between the numerical (generated by the algorithm) and experimental misalignment angle distributions.

\subsection{Generation of the 2D distribution}

A number of methods have been proposed to generate a 2D RVE for a given fibre volume fraction and distribution, e.g. Melro et al. [39], Vaughan and McCarthy [40]. The present study adopts the method proposed by Catalanotti [41] (later extended by Varandas et al. [42]) to generate the in-plane distribution of fibres in the matrix. This method is very flexible and can be used to generate a 2D distribution for any fibre volume fraction, $v_{f}$. In summary, the method generates the 2D RVE in three steps: (i) the generation of the compact RVE, the densest RVE with fibres arranged in a hexagonal configuration; (ii) the creation of the initial RVE, obtained by expanding the compact RVE to the desired dimensions; and finally, (iii) the generation of the final RVE, the distribution obtained after the fibres have been arranged randomly. Full details of the method are reported in [41]. The output of this step is a distribution of fibres in the matrix, with a prescribed $v_{f}$, and ensuring that adjacent fibres are not interpenetrating. In addition, geometrical periodicity is also included in the 2D RVE generation.

\section{2. $3 D$ randomly perturbed $R V E$}

The fibres are modeled as Bézier curves and misalignment is introduced by moving, in a random fashion, the control points. The analytical representation of the Bézier 


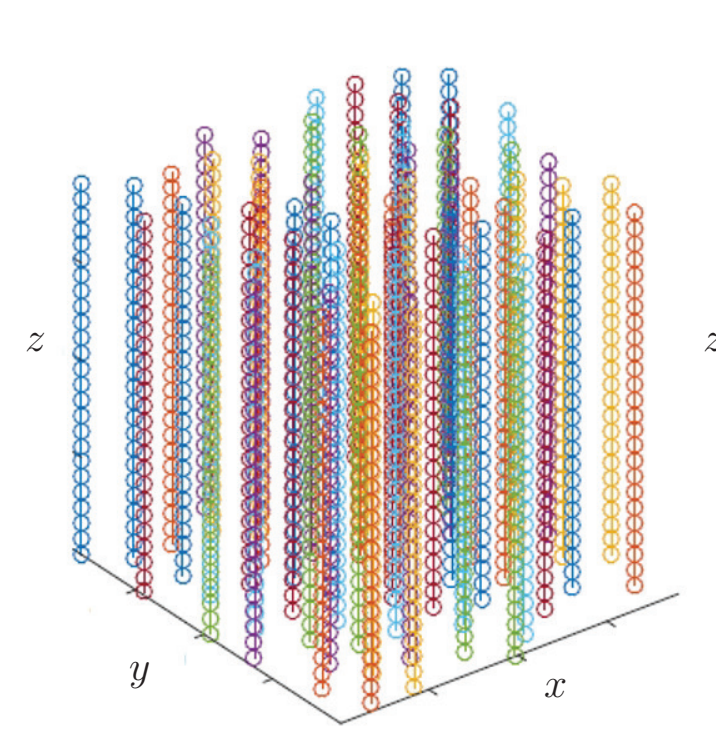

(a) Configuration with straight fibres

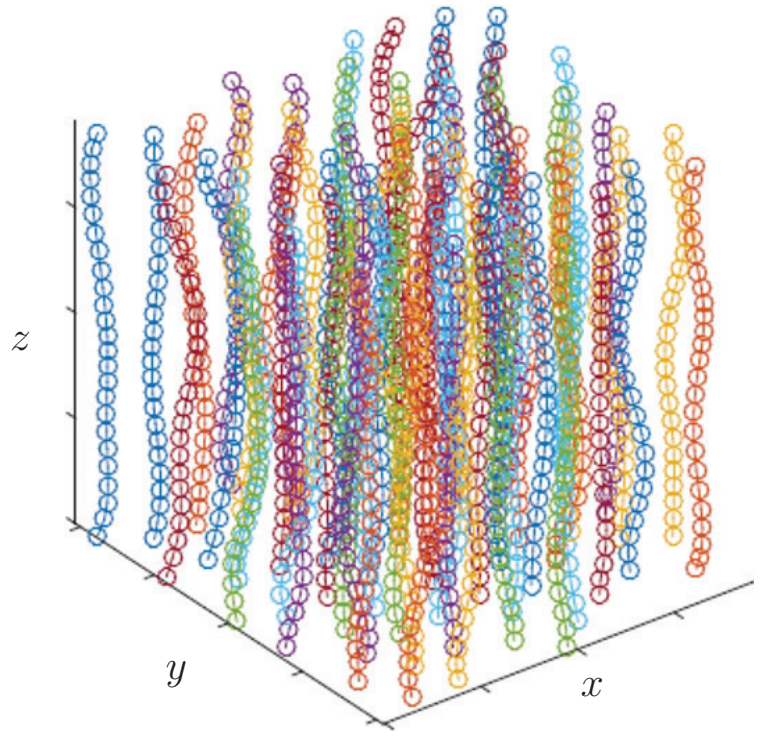

(b) Configuration after perturbation

Figure 3: Fiber misalignment through the perturbation process

curve is [43]:

$$
B(t)=\sum_{i=1}^{n} \frac{n !}{i ! \cdot(n-i) !} \cdot(1-t)^{n-i} \cdot t^{i} \cdot P_{i}
$$

where $n$ is the number of control points on each fibre, $P_{i}$ are the coordinates of the $i^{\text {th }}$ control point, and $t_{i}$ is a control variable with a value of 0 at the first control point and unity for the last control point.

The initial 2D RVE provides the position of each fibre that at the beginning are considered as perfectly straight. Therefore, the control points of each individual fibre have the same coordinates $x$ and $y$ (that have been identified by the 2D algorithm) and are equispaced along the $z$ direction, as shown in Figure 3(a). Fibre waviness is introduced by moving the control points on the plane perpendicular to $z$ in order not to change the $z$ coordinate of each control point. Repeating this operation for all the control points produce the perturbed configuration, as shown in Figure 3(b).

Therefore, the perturbation displacement, $\mathbf{u}$, applied to a given control point can be 
expressed as:

$$
\begin{aligned}
& u_{x}=\rho \cos \theta \\
& u_{y}=\rho \sin \theta \\
& u_{z}=0,
\end{aligned}
$$

where $\rho$ and $\theta$ are the coordinates of the control point in the polar coordinate system that lies on the plane perpendicular to $z$ and with the origin at the control point. The value of $\rho$ is calculated based on the distance between the current and the nearest fibre, $\bar{\rho}$. For a given value of $\bar{\rho}$, the value of the polar coordinate, $\rho$, is given by:

$$
\rho=\mathcal{R}_{1} \cdot \bar{\rho},
$$

where $\mathcal{R}_{1}$ is a random variable uniformed distributed in the interval $[0,1]$. The value of $\bar{\rho}$ is calculated as the value for which the fibre that is perturbed comes into contact with a neighbouring fibre. With this procedure, the perturbation of the control points cannot yield an intersection or contact between adjacent fibres. The angular coordinate, $\theta$, of the perturbation is random and is calculated as $\theta=2 \pi \mathcal{R}_{2}$, where $\mathcal{R}_{2}$ is a random variable uniformed distributed in the interval $[0,1]$. To ensure that the geometric periodicity of the RVE is maintained after the perturbation, the distance between two points on different fibres, with coordinates $p$ and $q$, is not simply given by the Euclidean distance $\sqrt{\sum_{j=1}^{3}\left(p_{j}-q_{j}\right)^{2}}$. Instead, for a rectangular prismatic RVE with a squared base of side $L$ and height $H$, the distance is calculated as:

$$
d=\sqrt{\sum_{j=1}^{3}\left(\min \left\{\left|p_{j}-q_{j}\right|, l_{j}-\left|p_{j}-q_{j}\right|\right\}\right)^{2}}
$$

where $p_{j}=\left\{p_{x}, p_{y}, p_{z}\right\}^{T}, q_{j}=\left\{q_{x}, q_{y}, q_{z}\right\}^{T}$, and $l_{j}=\{L, L, H\}^{T}$ [41]. The minimum distance between the points with coordinates $p$ and $q$ is also the distance between the fibres. Therefore, in order to calculate $\bar{\rho}$, the control point is incrementally moved, 
corresponding to an increment of $\rho$. When contact is detected $(\min (d)<2 R$; with $R$ is the fibre radius) the value assumed by $\rho$ corresponds to $\bar{\rho}$.

If in the RVE there are $n_{f}$ fibres, assuming that each fibre has $n$ control points, in the RVE there will be in total $n \cdot n_{f}$ control points. Therefore, this procedure is carried out $n \cdot n_{f}$ times for each iteration until the perturbed RVE is obtained (Figure $3(\mathrm{~b}))$. It is worth remarking that since the fibres are modelled as Bézier curves, the geometric description of the curve is provided only by the control points that can easily be exported and used to regenerate the curve in other environments. Moreover, the use of Bézier curves provides several advantages when imposing $C^{0}$ and $C^{1}$ periodicity since this can be enforced using the end point interpolation and the end point derivative properties of the Bézier curves, as explained in [30].

\subsection{Optimisation of the RVE}

The use of Equation 4 does not provide direct control over the fibre misalignment angle distribution. In order to produce statistically consistent RVEs, the misalignment angle distribution generated by the algorithm must match the empirically measured distribution. Hence, the radial coordinate $\rho$ is adjusted so that:

$$
\rho=\lambda \cdot \bar{\rho}
$$

where $\lambda$ is a parameter chosen in order to minimise the standard error between the numerical and experimental distribution. The standard error $(S E)$ can be defined in terms of likelihood or probability and always assume the form (see [30]):

$$
S E=S E(\lambda)
$$

Therefore, $\lambda$ is obtained by minimising Equation 7; this minimisation is carried out each time a control point is moved from its position. thus ensuring a close match between the constructed and measured fibre distributions. 


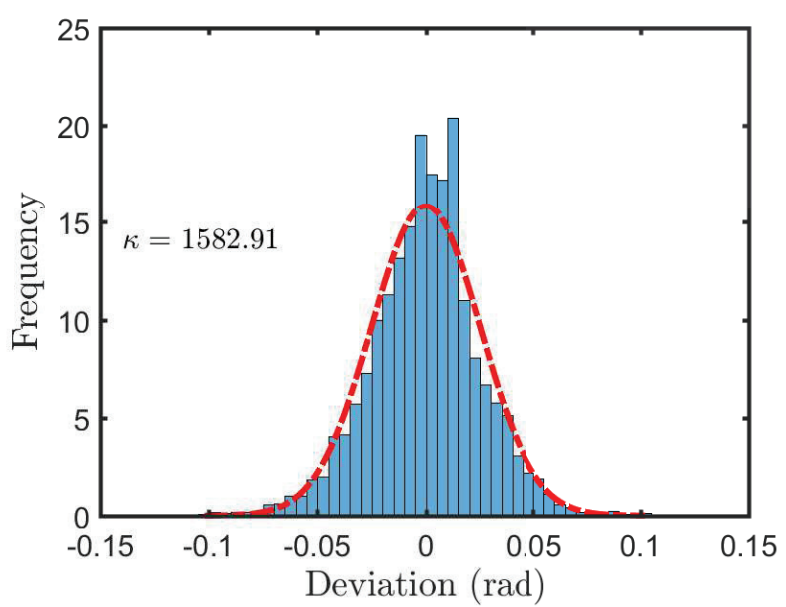

(a) IM7/8552

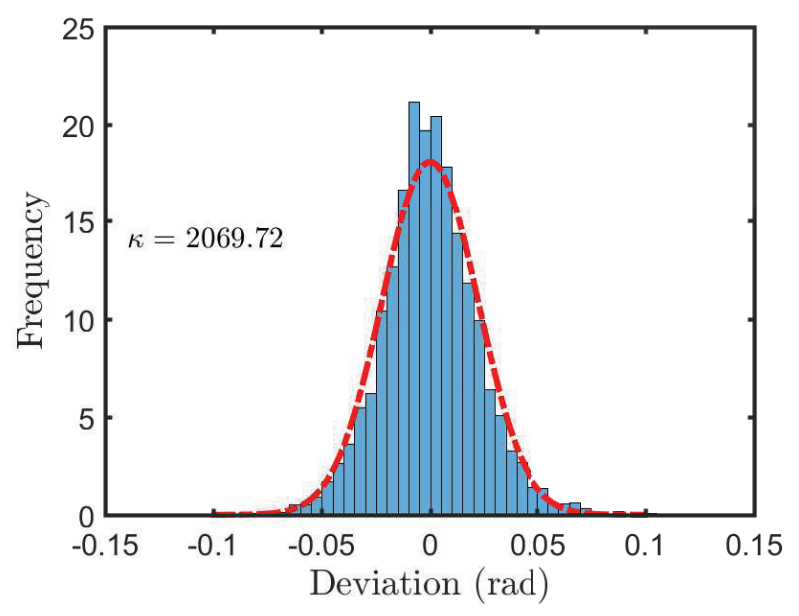

(b) IM7/PEEK

Figure 4: Fiber misalignment data for the two material system under consideration (the histogram represents the measurements and dotted line represents the von Mises distribution)

\section{Results and Discussion}

The procedure described in Sections 3 and 4 is applied to the two material systems discussed in Section 2. The measured fibre misalignments from the CT scans are shown in Fig. 4 for both material systems. Both histograms summarise the results of 4840 scans. The fibres are more aligned for the IM7/PEEK system Fig. 4(b), i.e., the misalignment angles are more concentrated around the mean value $\left(0^{\circ}\right)$. This results in smaller misalignment angles and a larger value of $\kappa$. For the IM7/8552 system, $\kappa=1583$, while for the IM7/PEEK system, $\kappa=2070$. During the manufacture of IM7/PEEK using ATP, solidification occurs while the fibres are subjected to tension and under outof-plane compression of the roller. This may explain the improved alignment for this material system. The misalignment in fibres for both cases ranges from $-5^{\circ}$ to $5^{\circ}$.

In [5] a fixed direction for neighbouring fibres (bundles) was proposed and in the current work this suggestion was confirmed in some cases. However, the general case shows that each fibre has its own independent orientation. Fig. 5 shows data collected for seven neighbouring fibres (F1-F7) in the same image. Over a very small area (300 $\mu \mathrm{m} \times 300 \mu \mathrm{m})$, the slope of each fibre segment is different. F4 represents an almost 


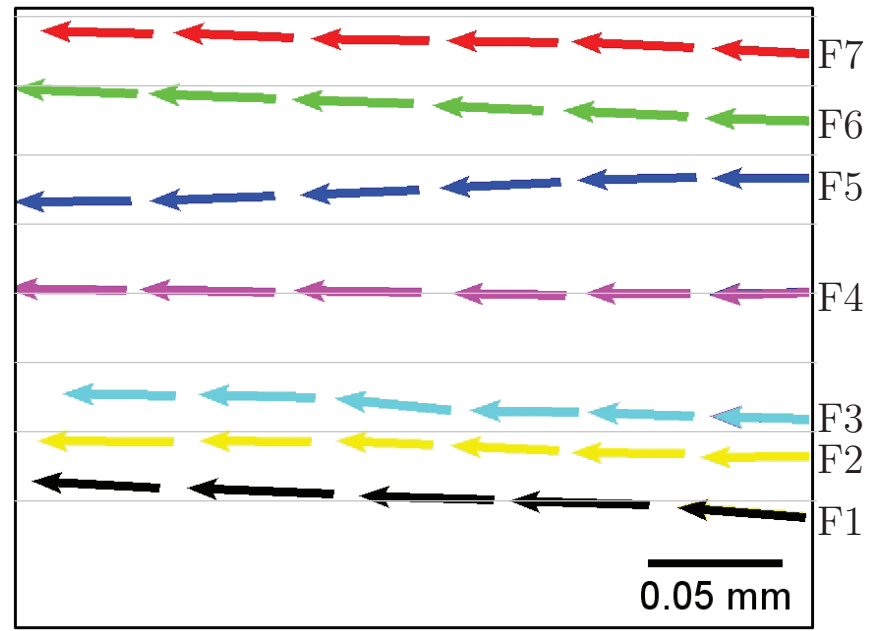

Figure 5: Fiber deviations as measured from a single 2D image (color codes represent individual fibres)

perfectly straight/horizontal fibre; F6 a fibre with almost constant slope along its length and F3 a fibre that shows an abrupt change of direction along its length. The other fibres show different slopes along their length. Based on these images, which are typical of the distributions we have examined, it was not possible to confirm that the misalignment follows a certain well-defined pattern, as proposed in [10, 12]. This implies that considering a misalignment with a defined wavelength and amplitude is not appropriate at this length scale. If it exists, the wavelength and amplitude of the fibre waviness is much larger than the length scale of the current study [12, 24].

The experimentally determined values of $\kappa$ were used with the algorithm described in Section 4. Fig. 6 shows the IM7/PEEK system with $\kappa=2070$ and $L=H=43 \mu \mathrm{m}$. It is worth remarking that the RVEs generated with the current model are flexible in terms of the in-plane dimensions $(L)$, the height to length ratio $(L / H)$ and the fibre volume fraction $\left(v_{f}\right)$. It may also be noted that the RVE constructed with this model is geometrically periodic, as guaranteed from the 2D model [41] and Eq. 5, and this may be noted from the distributions in Fig. 6.

To confirm that the algorithm provides correct fibre distributions, the fibre misalignments in both material systems are plotted in Fig. 7. The histogram represents the misalignment angles for the constructed fibres and the line represents the von Mises 


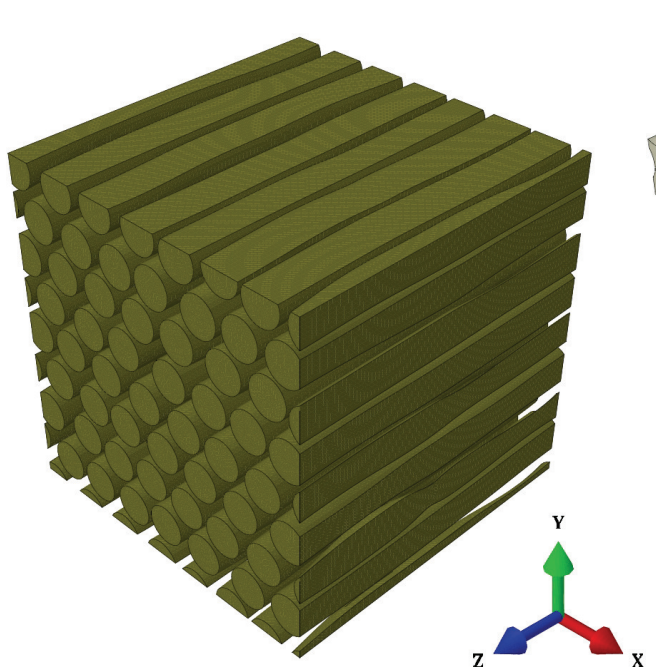

(a) Fibers

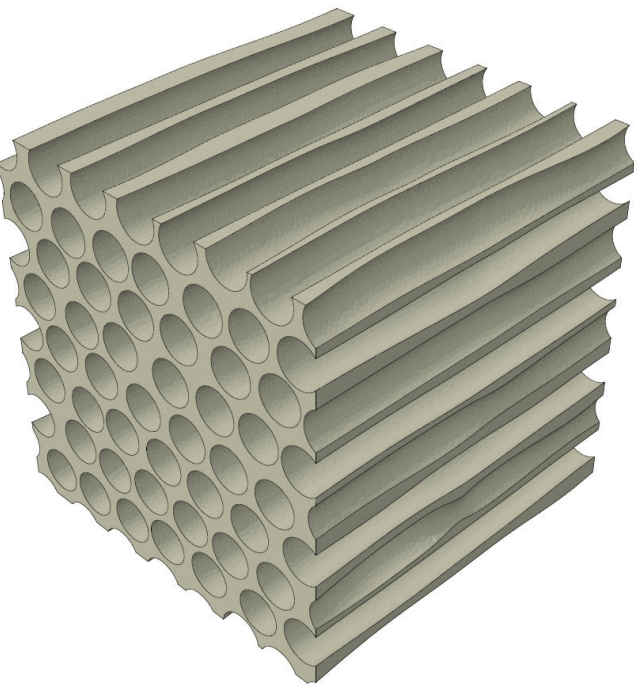

(b) Matrix

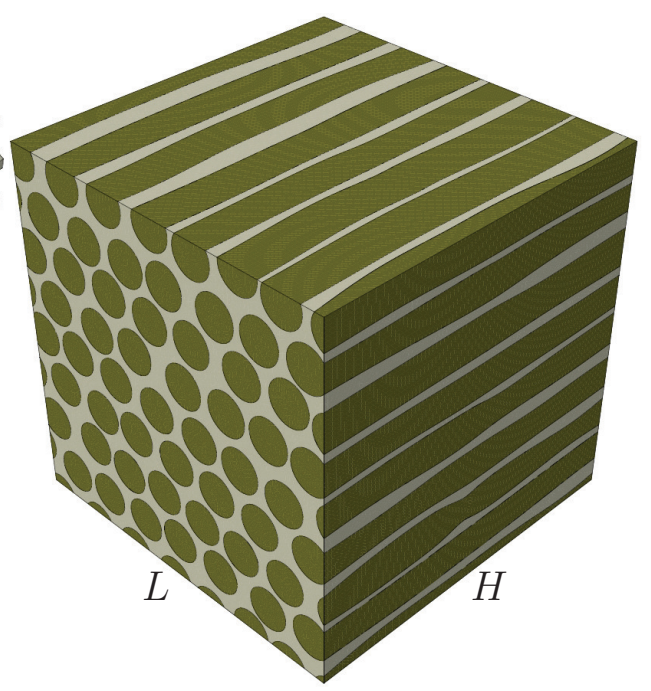

(c) Assembly of fibres and matrix

Figure 6: Constructed in the RVE for the IM7/PEEK

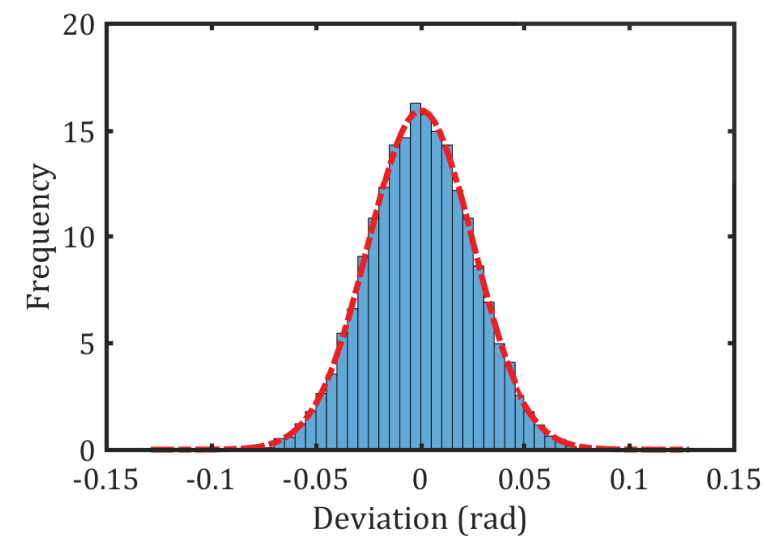

(a) $\operatorname{IM} 7 / 8552(\kappa=1583)$

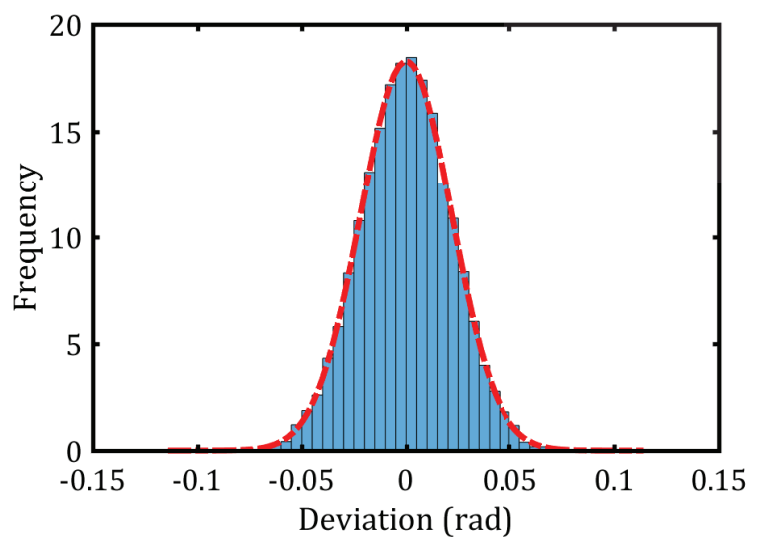

(b) $\operatorname{IM} 7 / \operatorname{PEEK}(\kappa=2070)$

Figure 7: Fiber misalignments in the constructed RVEs. The histogram represents the actual numerically constructed and thr red line represents the data measured experimentally)

distribution with the measured $\kappa$. Both sets of data are in very close agreement, which shows the capabilities of the current model to represent the experimentally measured fibre distribution.

Fig. 8 examines individual fibre geometries, constructed using the proposed method. It shows five fibres constructed in the same RVE. Minimum deviations are shown for F4 and F5 whereas, F2 and F3 show perfect alignment at a certain portion and then major 


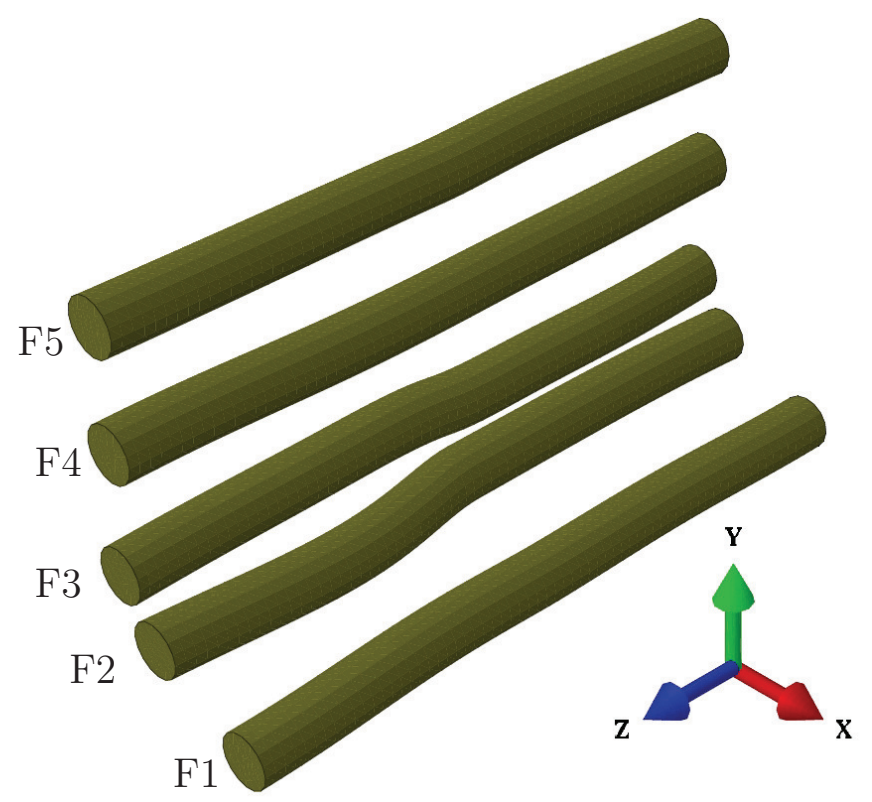

Figure 8: Fibre deviations as appearing in a constructed 3D RVE

deviations. A global buckling of the fibre can be seen in F1. In general, the features associated with the constructed fibres reflect the experimentally measured characteristics, as seen, for example, in Fig. 5. In future work, the effect of the model parameters (described in the previous paragraphs) on the accuracy of the predictions and the computational effort will be addressed.

\section{Conclusions}

In the work, fibre misalignment in fibre reinforced polymer composites was addressed for the first time through an integrated approach. The study examines two different material systems: IM7/8552 and IM7/PEEK, which have different trends in fibre misalignment. The method uses computed tomography (CT) scans to obtain a 3D image and images are processed using Imagej. Measured fibre misalignments are shown to be well represented by the von Mises distribution. The analysis has shown that the fibres in the IM7/PEEK system, manufactured by autoclave, are more aligned to the specified fibre direction, compared to the IM7/8552 system, manufactured using ATP.

From the modelling side, a 2D RVE is constructed following [41]. The centres of 
each fibre are extended in the $z$-direction and a set of control points is allocated along each fibre centreline at equal spacing, which are then perturbed randomly in the $x-y$ plane to change its coordinates. An optimization scheme were then applied to the RVE to minimise the difference between the von Mises concentration parameter measured experimentally and the one associated with the constructed RVE. The proposed method showed the capability to precisely model fibre misalignments in different material systems (based on the measured value of $\kappa$ ) providing a framework to study the influence of such misalignments on mechanical properties, for example through the use of finite element simulation.

\section{Acknowledgment}

This work has received funding from Enterprise Ireland (EI) and the European Union Horizon 2020 Research and Innovative Programme under the Marie Sklodowska-Curie grant agreement No. 713654. The technical assistance of Michael Byrne and David Jones of the School of Engineering at the University of Limerick is acknowledged.

\section{References}

[1] A. Bahmani, G. Li, T. L. Willett, and J. Montesano. Three-dimensional microscopic assessment of randomly distributed representative volume elements for high fiber volume fraction unidirectional composites. Composite Structures, 192:153-164, 2018.

[2] X. Bai, M. A. Bessa, A. R. Melro, P. P. Camanho, L. Guo, and W. K. Liu. Highfidelity micro-scale modeling of the thermo-visco-plastic behavior of carbon fiber polymer matrix composites. Composite Structures, 134:132-141, 2015.

[3] P. P. Parlevliet, H. E. N. Bersee, and A. Beukers. Residual stresses in thermoplastic composites - a study of the literature. Part III: effects of thermal residual stresses. Composites: Part A, 38:1581-1596, 2007. 
[4] S. S. Nimbal, M. M. Banker, A. Roopa, B. Varughese, and R. Sundaram. Effect of gap induced waviness on compressive strength of laminated composites. Materials Today: Proceedings, 4:8355-8369, 2016.

[5] W. B. Hillig. Effect of fiber misalignment on the fracture behavior of fiber-reinforced composites. Journal of Materials Science, 29:419-423, 1994.

[6] C. J. Creighton, M. P. F. Sutcliffe, and T. W. Clyne. A multiple field image analysis procedure for characterisation of fibre alignment in composites. Composites: Part A, 32:221-229, 2001.

[7] W. J. R. Christian, F. A. DiazDelaO, K. Atherton, and E. A. Patterson. An experimental study on the manufacture and characterization of in-plane fibre-waviness defects in composites. Royal Society Open Science, 5(180082), 2018.

[8] B. N. Fedulov, F. K. Antonov, A. A. Safonov, A. E. Ushakov, and S. V. Lomov. Influence of fibre misalignment and voids on composite laminate strength. Journal of Composite Materials, 49:2887-2896, 2015.

[9] D. Wilhelmsson, R. Gutkin, F. Edgren, and L. E. Asp. An experimental study of fibre waviness and its effects on compressive properties of unidirectional ncf composites. Composites: Part A, 107:665-674, 2018.

[10] P. Davidson, A. M. Waas, C. S. Yerramalli, K. Chandraseker, and W. Faidi. Effect of fiber waviness on the compressive strength of unidirectional carbon fiber composites. In $53^{r d} A I A A / A S M E / A S C E / A H S / A S C$ Structures, Structural Dynamics and Materials Conference, 2012.

[11] S. W. Yurgartis. Measurement of small angle fiber misalignments in continuous fiber composites. Composites Science and Technology, 30:279-293, 1987.

[12] M. R. Wisnom. The effect of fibre misalignment on the compressive strength of unidirectional carbon fibre/epoxy. Composites, 21:403-407, 1990. 
[13] D. P. Raghavalu Thirumalai, H. Lilholt, F. Aviles, T. Logstrup Andersen, and H. Knudsen. Fibre waviness and misalignment measurement of unidirectional glass/LPET commingled composites - Effect on mechanical properties. In the Riso International Symposium on Materials Science, volume 34, pages 349-363, 2013.

[14] S. Mukhopadhy, M. I. Jones, and S. R. Hallett. Compressive failure of laminates containing an embedded wrinkle; experimental and numerical study. Composites: Part A, 73:132-142, 2015.

[15] B. Larranaga-Valsero, R. A. Smith, R. B. Tayong, A. Fernandez-Lopez, and A. Guemes. Wrinkle measurement in glass-carbon hybrid laminates comparing ultrasonic techniques: A case study. Composites: Part A, 114:225-240, 2018.

[16] D. L. Shi, X. Q. Feng, Y. Y. Huang, K. C. Hwang, and H. Gao. The effect of nanotube waviness and agglomeration on the elastic property of carbon nanotubereinforced composites. Transactions of the ASME, 126:250-257, 2004.

[17] B. A. Bednarcyk, J. Aboudi, and S. M. Arnold. The effect of general statistical fiber misalignment on predicted damage initiation in composites. Composites: Part $B, 66: 97-108,2014$.

[18] A. S. Argon. Fracture of composites, Treatise on Materials Science and Technology, volume 1. Academic Press, INC, New York, 1972.

[19] J. Zhu, J. Wang, and L. Zu. Influence of out-of-plane ply waviness on elastic properties of composite laminates under uniaxial loading. Composite Structures, 132:440-450, 2015.

[20] T. Takeda. Micromechanics model for three-dimensional effective elastic properties of composite laminates with ply wrinkles. Composite Structures, 189:419-427, 2018.

[21] D. Pain and B. W. Drinkwater. Detection of fibre waviness using ultrasonic array scattering data. Journal of Nondestructive Evaluation, 32:215-227, 2013. 
[22] A. R. Clarke, G. Archenhold, and N. C. Davidson. A novel technique for determining the 3D spatial distribution of glass fibres in polymer composites. Composites Science and Technology, 55:75-91, 1995.

[23] K. K. Kratmann, M. P. F. Sutcliffe, L. T. Lilleheden, R. Pyrz, and O. T. Thomsen. A novel image analysis procedure for measuring fibre misalignment in unidirectional fibre composites. Composites Science and Technology, 69:228-238, 2009.

[24] K. Mizukami, Y. Mizutani, A. Todoroki, and Y. Suzuki. Detection of in-plane and out-of-plane fiber waviness in unidirectional carbon fiber reinforced composites using eddy current testing. Composites: Part B, 86:84-94, 2016.

[25] G. Requena, G. Fiedler, V. Seiser, P. Degischer, M. Di-Michiel, and T. Buslaps. 3DQuantification of the distribution of continuous fibres in unidirectionally reinforced composites. Composites: Part A, 40:152-163, 2009.

[26] M. P. F. Sutcliffe, S. L. Lemanski, and A. E. Scott. Measurement of fibre waviness in industrial composite components. Composites Science and Technology, 72:20162023, 2012.

[27] D. Wilhelmsson and L. E. Asp. A high resolution method for characterisation of fibre misalignment angles in composites. Composites Science and Technology, 165:214-221, 2018.

[28] Y. Li, B. Stier, B. Bednarcyk, J-W. Simon, and S. Reese. The effect of fiber misalignment on the homogenized properties of unidirectional fiber reinforced composites. Mechanics of Materials, 92:261-274, 2016.

[29] D. Wilhelmsson, R. Talreja, and L. E. Gutkin, R. Asp. Compressive strength assessment of fibre composites based on a defect severity model. Composites Science and Technology, 181(107685), 2019.

[30] G. Catalanotti and T. A. Sebaey. An algorithm for the generation of threedimensional statistically representative volume elements of unidirectional fibre- 
reinforced plastics: focusing on the fibres waviness. Composite Structures, 227(111272), 2019. doi: 10.1016/j . compstruct. 2019.111272.

[31] A. J. Comer, D. Ray, W. O. Obande, D. Jones, J. Lyons, I. Rosca, R. M. O'Higgins, and M. A. McCarthy. Mechanical characterisation of carbon fibre?peek manufactured by laser-assisted automated-tape-placement and autoclave. Composites: Part A, 69:10-20, 2015.

[32] D. Ray, A. J. Comer, J. Lyons, W. Obande, D. Jones, R. M. O'Higgins, and M. A. McCarthy. Fracture toughness of carbon fiber/polyether ether ketone composites manufactured by autoclave and laser-assisted automated tape placement. Journal of Applied Polymer Science, (41643), 2015.

[33] C. A. Schneider, W. S. Rasband, and K. W. Eliceiri. NIH image to ImageJ: 25 years of image analysis. Nature Methods, 9:671-675, 2012.

[34] P. M. Jelf and N. A. Fleck. The failure of composite tubes due to combined compression and torsion. Journal of Materials Science, 29:3080-3084, 1994.

[35] E. Van Doorn, B. Dhruva, K. R. Sreenivasan, and V. Cassella. Statistics of wind directions and its increments. Physics of Fluids, 12:1529-1534, 2000.

[36] P. Berens. CircStat: A MATLAB toolbox for circular statistics. Journal of Statistical Software, 31:1-21, 2009.

[37] Y. Li, J.W. Simon, and S. Reese. Microsphere-based homogenization for unidirectional fiber reinforced composites. In $5^{\text {th }}$ GACM colloquium on computational mechanics, 2013.

[38] S. R. Jammalamadaka and A. SenGupta. Topics in Circular Statistics. World Scientific Press, Singapore, 2001.

[39] A. R. Melro, P. P. Camanho, and S. T. Pinho. Generation of random distribution 
of fibres in long-fibre reinforced composites. Composites Science and Technology, 68:2092-2102, 2008.

[40] T. J. Vaughan and C. T. McCarthy. Micromechanical modelling of the transverse damage behaviour in fibre reinforced composites. Composites Science and Technology, 71:388-396, 2011.

[41] G. Catalanotti. On the generation of RVE-based models of composites reinforced with long fibres or spherical particles. Composite Structures, 138:84-95, 2016.

[42] L. F. Varandas, A. Arteiro, M. A. Bessa, A. R. Melro, and G. Catalanotti. The effect of through-thickness compressive stress on mode II interlaminar crack propagation: A computational micromechanics approach. Composite Structures, 182:326-334, 2017.

[43] G. E. Farin, J. Hoschek, and M. S. Kim. Handbook of Computer Aided Geometric Design. Elsevier Science B.V., Amsterdam, The Netherlands, 2002. 